\title{
Altered Polarization, Morphology, and Impaired Innate Immunity Germane to Resident Peritoneal Macrophages in Mice with Long-Term Type 2 Diabetes
}

\author{
Hui-Fang Liu, ${ }^{1}$ Hui-Jie Zhang, ${ }^{1}$ Qi-Xian Hu, ${ }^{1}$ Xiao-Yan Liu, ${ }^{1}$ \\ Zhi-Quan Wang, ${ }^{2}$ Jia-Yan Fan, ${ }^{3}$ Ming Zhan, ${ }^{2}$ and Feng-Ling Chen ${ }^{1}$ \\ ${ }^{1}$ Department of Endocrinology, The Shanghai Third People's Hospital, Shanghai Jiao Tong University School of Medicine, \\ 280 Mohe Road, Shanghai 201900, China \\ ${ }^{2}$ State Key Laboratory of Medical Genomics, Ruijin Hospital, Shanghai Jiao Tong University School of Medicine, \\ 197 Ruijin No. 2 Road, Shanghai 200025, China \\ ${ }^{3}$ Department of Ophthalmology, The Shanghai Ninth People's Hospital, Shanghai Jiao Tong University School of Medicine, \\ 639 Zhizaoju Road, Shanghai 200011, China
}

Correspondence should be addressed to Feng-Ling Chen, fengling_chen@hotmail.com

Received 17 March 2012; Revised 13 June 2012; Accepted 29 June 2012

Academic Editor: Soldano Ferrone

Copyright (C) 2012 Hui-Fang Liu et al. This is an open access article distributed under the Creative Commons Attribution License, which permits unrestricted use, distribution, and reproduction in any medium, provided the original work is properly cited.

Type 2 diabetes (T2D) is associated with perturbed innate immunity. Macrophages, bridging innate immunity and metabolic disturbances, play important roles in controlling immune homeostasis. However, the effect of long-term diabetic milieu (DM) on the functions and phenotypes of macrophages is still not clear. In this study, we used resident peritoneal macrophages (RPMs) from 5 -month-old $\mathrm{db} / \mathrm{db}$ mice to investigate the changes of macrophages. It was found that RPMs in $\mathrm{db} / \mathrm{db}$ mice significantly reduced phagocytosis and adhesion capacity. After standardization with body weight, the number of F4/80 $0^{+} \mathrm{RPMs}$ markedly reduced in $\mathrm{db} / \mathrm{db}$ mice, and, furthermore, the macrophages skewed to M2-polarizated macrophages. The results of morphology found that the RPMs shape of $\mathrm{db} / \mathrm{db}$ mice was nearly round, but the RPMs shape of control mice was spindle-shaped and irregular. In this study, we found the cell numbers, morphology, and innate immunity functions of RPMs in 5-month-old type 2 diabetic mice ( $\mathrm{db} / \mathrm{db}$ mice) obtained by abdominal cavity lavage were significantly altered. Importantly, we also found the remarkably increased M2-RPMs in diabetic mice for the first time.

\section{Introduction}

Type 2 diabetes (T2D) is characterized by hyperglycemia and dyslipidemia resulting from a combination of insulin resistance in the skeletal muscle, liver, and adipose tissues, and impaired insulin secretion from the pancreatic islets, which is putatively due to impaired immune response [1]. Patients with T2D exhibit increased susceptibility to infection, impaired wound healing, and other inflammatory or degenerative manifestations [2]. It is the macrophage system dysfunction that leads to the major alterations among these innate immune abnormalities $[3,4]$. Macrophage system including circulating monocytes, tissue macrophages, and dendritic cells is characterized by a high level of plasticity and widespread tissue distribution. It was observed that phagocytes in type 2 diabetic patients were in a heightened state of oxidative stress accompanied by impaired bactericidal activity and chemotaxis [5-7]. Furthermore, the receptor functions, levels of cytokines expression, and release of the macrophages derived from the peripheral blood of T2D patients all changed dramatically [8-10].

Monocytes derived from bone marrow remain in the blood circulation system for 1-2 days before they migrate into the peripheral tissues, where they differentiate into fully mature resident macrophages. In innate immunity, resident macrophages provide immediate defense against foreign pathogens and assist leukocyte infiltration [11]. Resident macrophage population is composed of various cells, including macrophages in the peritoneal cavity, which is thought fully developed and adaptable to their local microenvironment with a wide diversity in terms of their function and phenotype $[12,13]$. The evidence that macrophages could 
display different activation states with distinct properties provided us with clues to understand diverse functions of macrophages [14]. Two polarized states in activated macrophages have been defined, the pro-inflammatory, classically activated state M1 induced by type I cytokines (e.g., TNF) or bacterial products (e.g., LPS) and the antiinflammatory, alternatively activated phenotype M2 induced by IL-4, IL-10, or glucocorticoid hormones $[15,16]$. These different phenotypes of macrophages were expressed in such a manner that macrophages display a balanced, integrated pattern of functions [14, 17].

Most of the current understanding of macrophage biology came from analyses of cells drawn from the mouse peritoneal cavity, which became widely used as a macrophage source as early as the 1960s [18]. A recent study reported marked difference between resident and induced macrophages, such as the efficacy of killing pathogenic microorganisms and antigen presentation [19]. Furthermore, studies about alteration of macrophage characteristics in diabetes were performed in streptozotocin- (STZ-) induced type 1 diabetic mouse model, which was accompanied by immunological dysfunction [20]. It is hard to determine whether the dysfunctions of macrophages in these diabetes hosts were solely caused by diabetes itself or just by the host immunological dysfunction. To the best of our knowledge, little is known about the polarized states of RPMs.

To provide evidence showing the effects of DM with long duration on cell numbers, morphology, and innate immunity functions of macrophages in vivo, we used resident peritoneal macrophages (RPMs) from 5-month-old $\mathrm{db} / \mathrm{db}$ mice obtained by abdominal cavity lavage to study the changes. The result may help us to understand the susceptibility of diabetic patients to infectious diseases and other complications in the late stage of diabetes.

\section{Materials and Methods}

2.1. Reagents. Ethylenediaminetetraacetic acid (EDTA), Nformyl-L-methionyl-L-leucyl-L-phenylalanine (FMLP), paraformaldehyde, modified Wright-Giemsa stain solution, and fluorescent red, carboxylate-modified polystyrene latex beads were purchased from Sigma-Aldrich (St. Louis, MO, USA). RPMI-1640, fetal bovine serum (FBS), penicillin/streptomycin, and phosphate buffered saline (PBS) were obtained from Gibco BRL (Grant Island, NY, USA). Bovine serum albumin (BSA) was ordered in BD Pharmingen (San Diego, CA, USA). Beyotime Institute of Biotechnology provided crystal violet staining solution (Shanghai, China).

2.2. Animals. Eight-to-twelve-week-old male $\mathrm{db} / \mathrm{db}$ (C57BL/ 6J-Lepr ${ }^{\mathrm{db}} / \mathrm{Lepr}^{\mathrm{db}}$ ) mice and littermate controls (C57BL/6J background) were fed chow (LabDiet 5001) and bought from the Model Animal Research Center of Nanjing University (Nanjing, China). All mice were kept in a specific pathogenfree facility and given free access to food and water. The experiment was performed strictly in accordance with the institutional guidelines for the care and use of laboratory animals of Shanghai Jiao Tong University.

2.3. Isolation and Culture of Mouse RPMs. Mouse RPMs were obtained by the modified method of Cohn and Benson [21]. Approximately $6 \mathrm{~mL}$ cold serum-free RPMI-1640 medium was injected intraperitoneally after mice were killed under ether anesthesia. After a gentle massage, peritoneal fluid containing cells was harvested and centrifuged at $700 \mathrm{~g}$ for $5 \mathrm{~min}$ at $4{ }^{\circ} \mathrm{C}$. The pellet was resuspended with $5 \mathrm{~mL}$ of RPMI1640 containing $10 \%$ FCS, 100 units $/ \mathrm{mL}$ penicillin, and $100 \mu \mathrm{g} / \mathrm{mL}$ streptomycin and cultured at $37^{\circ} \mathrm{C}$ in a $35 \mathrm{~mm}$ dish (Corning, NY, USA) for one hour, and only attached macrophages were used for the following experiments. Adherent cells were collected with $5 \mathrm{~mm}$ EDTA (SigmaAldrich) in ice-cold PBS (Gibco BRL). The cell viability was more than $90 \%$, and more than $90 \%$ of adherent cells were $\mathrm{F} 4 / 80^{+}$macrophages, as reported previously $[22,23]$.

2.4. Morphology Observation. RPMs from $\mathrm{db} / \mathrm{db}$ or control mice $\left(1 \times 10^{5}\right)$ were cultured in 24-well plates containing $12 \mathrm{~mm}$ sterilized glass slides per well for $2 \mathrm{~h}$. For microscopic studies, the adherent cells were stained by modified WrightGiemsa stain (Sigma-Aldrich) according to manufacturer's protocol. The glass slides were rinsed briefly in running deionized water (PH7.2) and dried in air thoroughly before capturing images by a microscope (Olympus BX51, Olympus Optical, Tokyo, Japan).

2.5. Immunofluorescence Staining and Laser Confocal Scanning Microscopy. RPMs from db/db or C57BL/6J mice were collected then cultured on 24-well plates containing $12 \mathrm{~mm}$ glass slides at a concentration of $2 \times 10^{5}$ macrophages per well for $2 \mathrm{~h}$. The adherent macrophages were fixed in $4 \%$ paraformaldehyde (Sigma-Aldrich) for $20 \mathrm{~min}$ then incubated in PBS with $0.5 \% \mathrm{BSA}$ at $4^{\circ} \mathrm{C}$ for $30 \mathrm{~min}$ in order to avoid nonspecific binding to FcR. Primary antibodies against F4/80 (EMR1: R\&D Systems, USA) and Cy3-labeled second antibodies (Beyotime Institute of Biotechnology, Shanghai, China) were sequentially incubated with macrophages for overnight at $4^{\circ} \mathrm{C}$ and $2 \mathrm{~h}$ at room temperature, respectively. After DAPI (Invitrogen, San Diego, CA, USA) staining, cells were analyzed under laser confocal scanning microscopy (LSM510, Zeiss, Germany).

2.6. Immunofluorescence Staining and Flow Cytometry. At least $5 \times 10^{5}$ RPMs were incubated with $2.4 \mathrm{G} 2$ (BD Biosciences) for $10 \mathrm{~min}$ at room temperature then with primary antibodies or the matching control isotypes for $30 \mathrm{~min}$ at $4^{\circ} \mathrm{C}$. Thereafter, the cells were rinsed twice and resuspended in Pharmingen stain buffer (BD Biosciences) for assay by FACScalibur flow cytometer (Becton Dickinson, Franklin Lakes, NJ). The data were analyzed with Cell Quest software (Becton Dickinson, Mountain, CA, USA). For three-color staining, RPMs were stained with phycoerythrinCy5 (PE-Cy5-) labeled anti-F4/80 monoclonal antibodies (mAbs) (eBiosciences, San Diego, CA, USA), phycoerythrin (PE-) labeled anti-CD40 (eBiosciences) mAbs, or fluorescein 


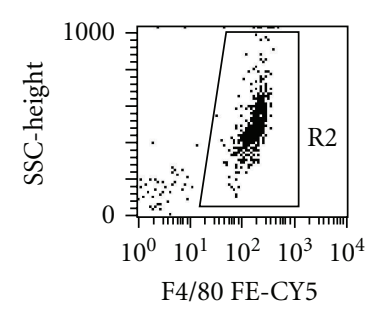

(a)

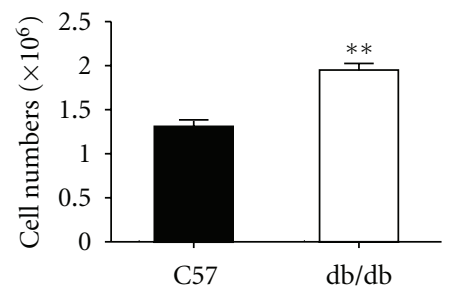

(b)

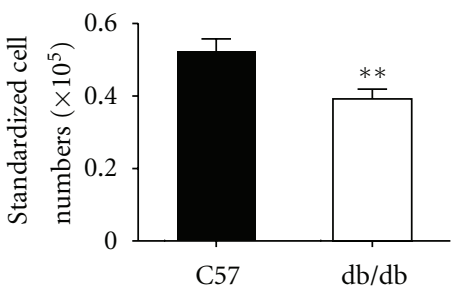

(c)

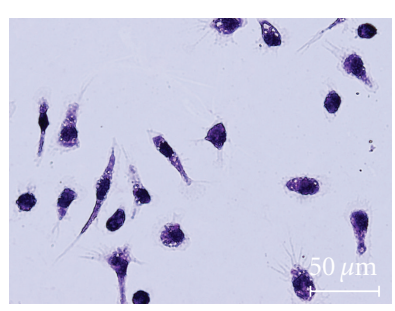

(d)

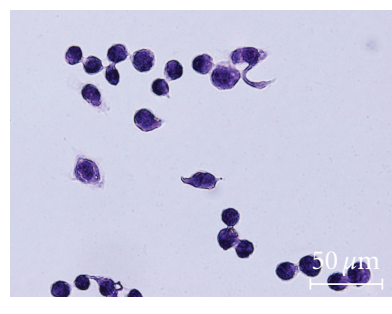

(e)

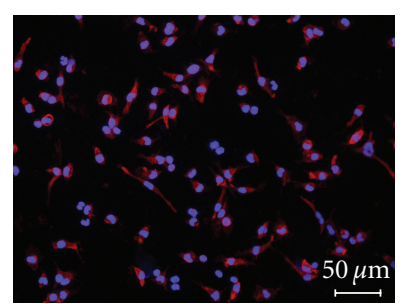

(f)

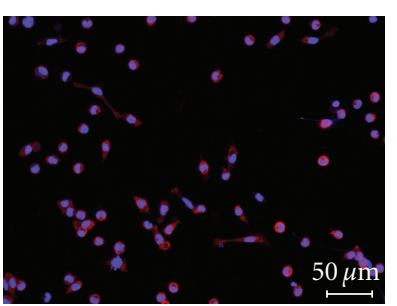

(g)

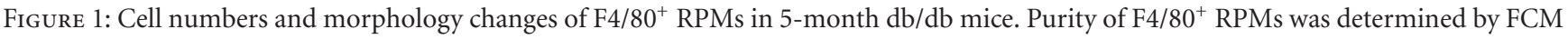
(a). The number of F4/80 RPMs was calculated by multiplying the total number of trypan blue negative cells by the ratio of F4/80 positive cells. The average number of RPMs of six $\mathrm{db} / \mathrm{db}$ or control mice was caculated (b), and the mean value of standardized RPM numbers with body weight from six db/db or control mice was taken (c). Morphology of RPMs was assayed by Wright-Giemsa staining or doubleimmunofluorescence staining and laser confocal scanning microscopy. RPMs from control mice (d) and diabetic mice (e) were stained by Wright-Giemsa; (f) and (g) were F4/80 RPMs from control or diabetic mice, respectively, assayed by double-immunofluorescence staining and laser confocal scanning microscopy. Scale bars represent $50 \mu \mathrm{m} .{ }^{* *} P<0.01$ between the indicated groups.

isothiocyanate (FITC-) labeled anti-CD206 mAbs (BioLegend, San Diego, CA, USA) for $30 \mathrm{~min}$ at $4^{\circ} \mathrm{C}$. M1 and M2 macrophages were identified as $\mathrm{F} 4 / 80^{+} / \mathrm{CD} 40^{+} / \mathrm{CD} 206^{-}$and $\mathrm{F} 4 / 80^{+} / \mathrm{CD} 40^{-} / \mathrm{CD} 206^{+}$cells, respectively.

2.7. Phagocytosis Assay. RPMs $\left(4 \times 10^{5}\right)$ were seeded in a 6well plate then incubated with fluorescent red, carboxylatemodified polystyrene latex beads $\left(8 \times 10^{6}\right.$ beads $)$ for $2 \mathrm{~h}$ at $37^{\circ} \mathrm{C}$, while the control were incubated at $4^{\circ} \mathrm{C}$. The cells were washed with PBS before harvest and then resuspended in PBS containing 5\% BSA. The phagocytic activity of RPMs was determined by measuring phagocytic rate and mean fluorescence intensity (MFI) with FACScalibur flow cytometer (Becton and Dickinson).

2.8. Adherence Assay. The adherence capacity of RPMs was evaluated by the adherence index (AI), capacity of adherent to plastic surfaces [24]. Briefly, $500 \mu \mathrm{L}$ RPMI-1640 complete medium containing $7.5 \times 10^{5} \mathrm{RPMs}$ was placed in each Eppendorf tube, incubated at $37^{\circ} \mathrm{C}$ with $5 \% \mathrm{CO}_{2}$ in a shaking bath for 10,30 , or $60 \mathrm{~min}$, respectively. AI was calculated according to the equation: $\mathrm{AI}=100-$ (number of macrophages ( $\mathrm{mL}$ supernatant $)^{-1} /$ number of macrophages $\left.(\mathrm{mL} \text { original sample })^{-1}\right) 100$.

2.9. Chemotaxis Assay. Chemotaxis experiment was carried out in $6.5 \mathrm{~mm}$ polycarbonate membrane inserts (Costar, Cambridge, MA, USA) with a $5 \mu \mathrm{m}$ pore size. Briefly, $100 \mu \mathrm{L}$ RPMs suspension $\left(1.3 \times 10^{5} /\right.$ well $)$ was deposited in the insert and $600 \mu \mathrm{L}$ RPMI-1640 containing FMLP (Sigma-Aldrich) $(0.1 \mathrm{mmol})$ was added into the lower compartment to induce chemotaxis for $2 \mathrm{~h} \mathrm{[25].} \mathrm{After} \mathrm{the} \mathrm{nonmigrated} \mathrm{cells} \mathrm{were}$ removed from the polycarbonate membrane upper side, the migrated cells which were on the lower side were fixed and stained by crystal violet staining solution (Beyotime Institute of Biotechnology). The chemotactic index (CI) is presented as the total number of migrated cells of 16 random selected microscopic fields (at $\times 400$ magnification).

2.10. Statistical Analysis. Data were expressed as means \pm standard deviation (SD). Student's unpaired $t$-test for comparison of means was used to compare means of each group. Differences were considered statistically significant at $P<$ 0.05 .

\section{Results}

3.1. Significantly Decreased Cell Numbers and Altered Cellular Morphology of F4/80 $0^{+}$RPMs in 5-Month-Old db/db Mice. To determine the long effect of T2D on cell numbers and cellular morphology of RPMs in $\mathrm{db} / \mathrm{db}$ mice, we identified resident macrophages by detecting the surface antigens F4/80 firstly. The purity of mouse $\mathrm{F} 4 / 80^{+} \mathrm{RPMs}$ was routinely determined by FCM before each assay, which was more than $90 \%$ (Figure 1(a)). As shown in Figure 1(b), the total cell numbers of $\mathrm{F} 4 / 80^{+} \mathrm{RPMs}$ in each $\mathrm{db} / \mathrm{db}$ mouse were far greater than that of control $(P<0.01, n=6)$. However, after the data were standardized with body weight, the result of RPM numbers reversed (Figure 1(c)). The morphology of RPMs was determined by Wright-Giemsa stain and a two-photon laser scanning. As shown in Figures 1(e) and 1(g), freshly isolated $\mathrm{F} 4 / 80^{+} \mathrm{RPMs}$ from $\mathrm{db} / \mathrm{db}$ mice were nearly round 


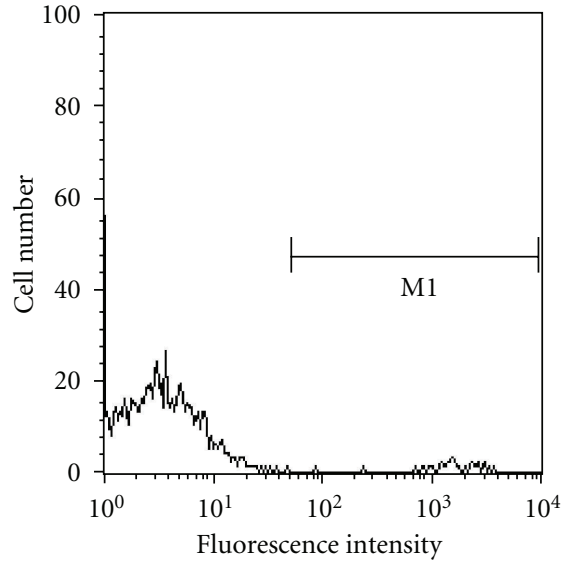

(a)

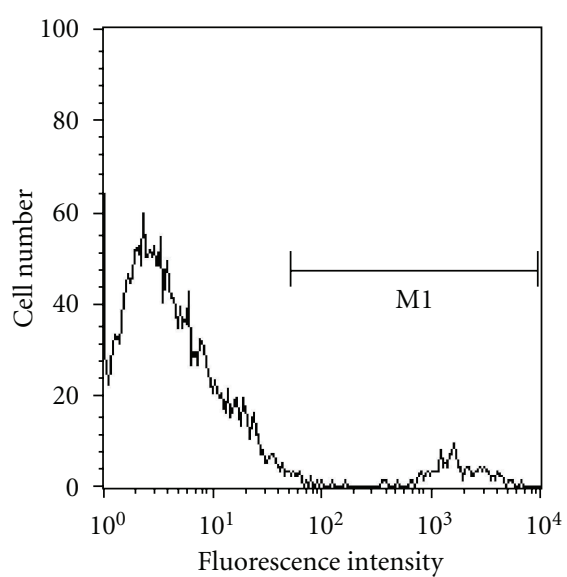

(c)

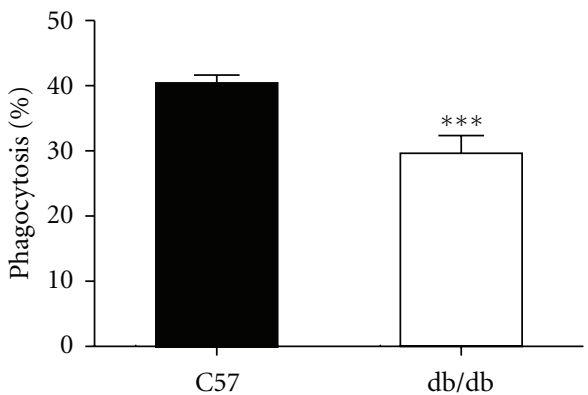

(e)

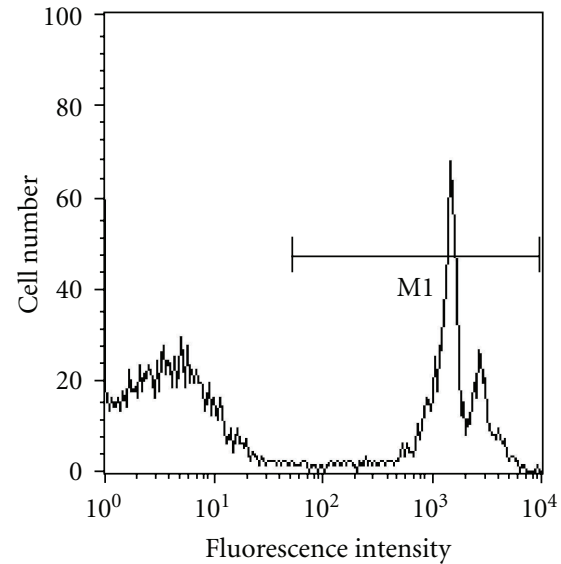

(b)

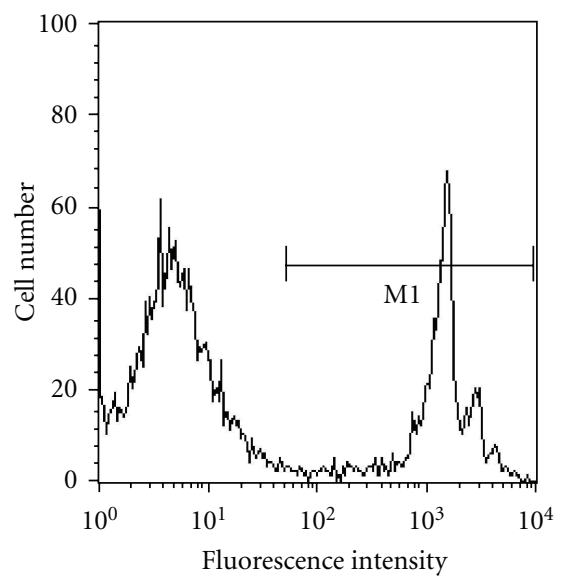

(d)

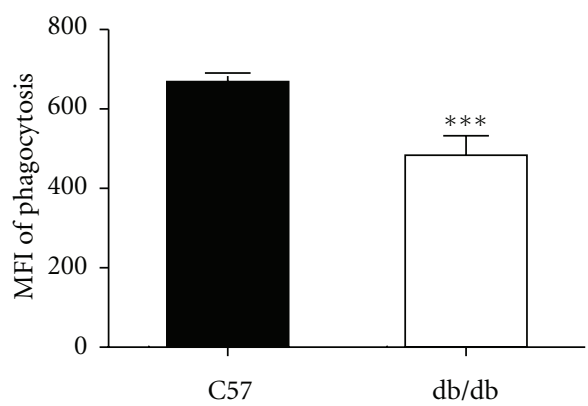

(f)

Figure 2: Decreased phagocytosis of polystyrene latex beads by $\mathrm{F} 4 / 80^{+} \mathrm{RPMs}$ in 5 -month diabetic mice. One representative experiment of 4 detected by FCM showing RPMs from normal mice (b) and db/db mice (d) after incubated with fluorescence-modified polystyrene latex beads for $2 \mathrm{~h}$ at $37^{\circ} \mathrm{C}$, wheals the control from normal mice (a) and $\mathrm{db} / \mathrm{db}$ mice (c) were incubated at $4^{\circ} \mathrm{C}$. The average phagocytic rate (e) and MIF (f) of four independent experiments were quantified. ${ }^{* * *} P<0.001$ between the indicated groups.

shape, while that from control mice displayed spindle-shaped or irregular morphology (Figures $1(\mathrm{~d})$ and $1(\mathrm{f})$ ). Thus, a significant alteration of cell numbers and morphology of $\mathrm{F} 4 / 80^{+}$ RPMs occurred in the mice with a long duration of DM.

\subsection{Nonspecific Phagocytosis Deficiency of $F 4 / 80^{+}$RPMs from} 5-Month-Old db/db Mice. Phagocytosis represents an early and crucial event in triggering host defenses against invading pathogens. The nonspecific phagocytic capacity of $\mathrm{F} 4 / 80^{+}$ RPMs against fluorescence-modified polystyrene latex beads was assessed by using FCM and shown in Figure 2. As compared with mice in the control group (Figure 2(b)), cell-related fluorescence intensity corresponding to the peak of the histograms moved to the left in $\mathrm{db} / \mathrm{db}$ mice, which indicated that fewer fluorescent beads were ingested by macrophages (Figure $2(\mathrm{~d})$ ). Both the phagocytic rate 


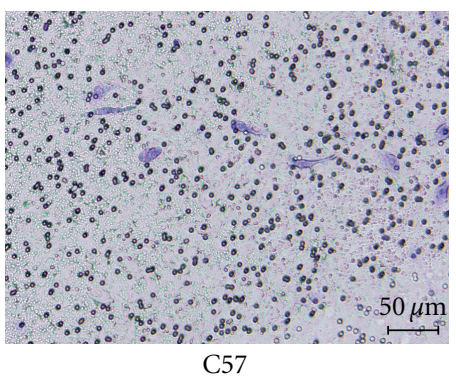

(a)

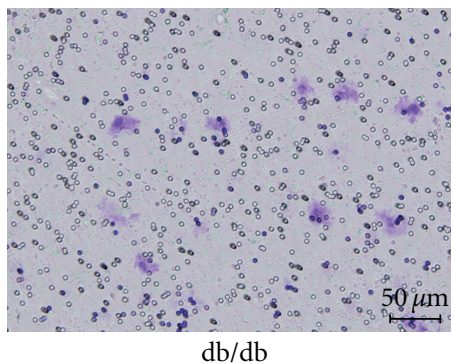

(c)

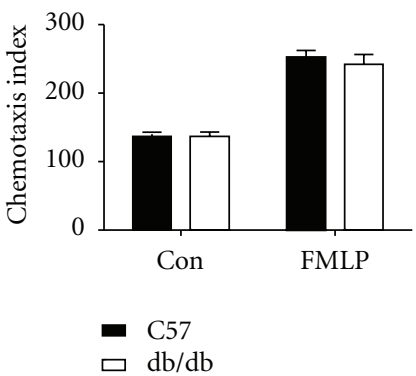

(e)

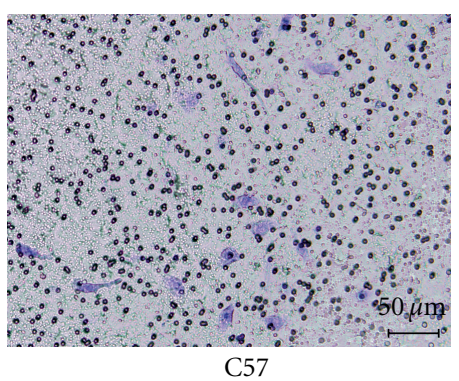

(b)

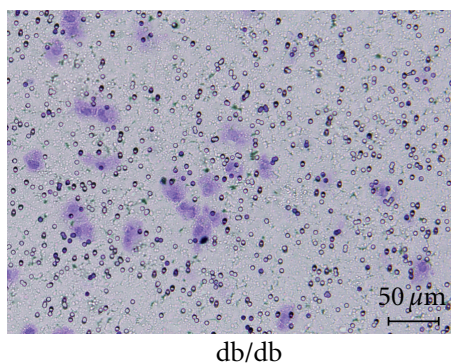

(d)

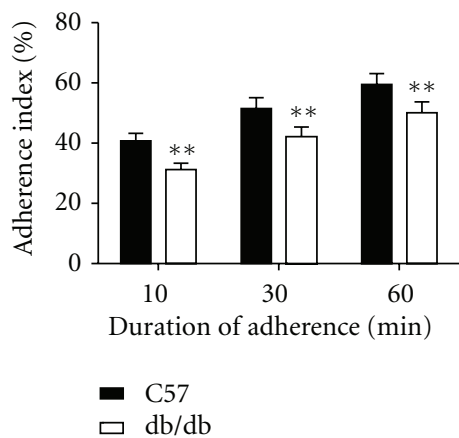

(f)

FIGURE 3: Impaired chemotaxis and adhesion capacity of RPMs from 5-month $\mathrm{db} / \mathrm{db}$ mice. FMLP induced migration of RPMs from normal mice (b) and db/db mice (d) was stained by Crystal Violet; while (a) and (c) were RPMs from normal and db/db mice, respectively, with no chemokine by crystal violet staining. The average CI (e) and AI (f) of RPMs of four independent experiments was quantified. Scale bars represent $50 \mu \mathrm{m} .{ }^{* *} P<0.01$ versus the control group.

(Figure 2(e), $P<0.001, n=4)$ and MFI of RPMs from $\mathrm{db} / \mathrm{db}$ mice were much lower than those of control group (Figure 2(f), $P<0.001, n=4$ ).

\subsection{Impaired Adhesion Vitality and Chemotaxis Capacity of} F4/80 ${ }^{+}$RPMs in 5-Month-Old db/db Mice. To further determine other functional changes like adhesion and chemotaxis in macrophage from mice with a long duration of diabetes, we investigated the chemotaxis and adhering capacity of RPMs. It was showed in Figures 3(b) and 3(d) that RPMs from control or $\mathrm{db} / \mathrm{db}$ mice migrated to the lower side of the filter after exposure to $0.1 \mathrm{mmol}$ FMLP for $2 \mathrm{~h}$ by crystal violet staining. The values of CI of RPMs in diabetic mice were lower than those of control without significant difference (Figure 3(e), $P>0.05, n=4)$. The adhering capacity of RPMs was assayed at selected time points (10 min,
$30 \mathrm{~min}$, and $60 \mathrm{~min})$, respectively, after macrophages were placed in each eppendorf tube for a shaking bath as described in Section 2. As shown in Figure 3(f), the values of AI of RPMs from $\mathrm{db} / \mathrm{db}$ mice were all significantly lower than those of the controls $(P<0.01, n=4)$.

\subsection{Phenotype Changes of F4/80 $0^{+}$RPMs in 5-Month-Old} $\mathrm{db} / \mathrm{db}$ Mice. The expression of CD40 on macrophages after activation is critically related to the efficient promotion of adaptive immunity by macrophages. CD206 expression is an important marker for IL-4-induced M2 macrophages [26]. Thus, we detected CD40 and CD206 expressions on RPMs from $\mathrm{db} / \mathrm{db}$ or control mice by FCM to study the polarized state as described in Section 2. As shown in Figure 4(c), the percentage of $\mathrm{M} 2\left(\mathrm{~F} 4 / 80^{+} / \mathrm{CD} 40^{-} / \mathrm{CD} 206^{+}\right)$macrophages $(P<0.01, n=4)$ of RPMs in $\mathrm{db} / \mathrm{db}$ mice was significantly 


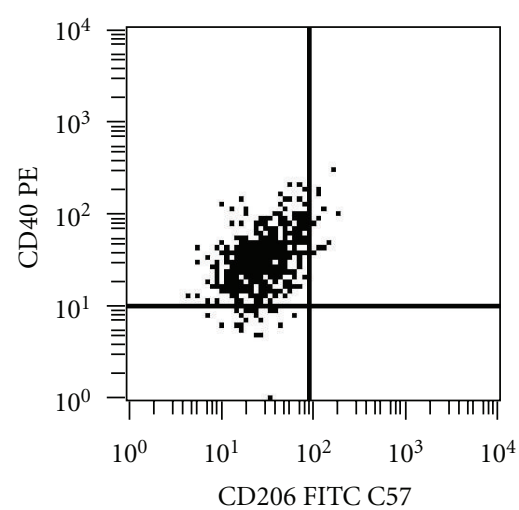

(a)

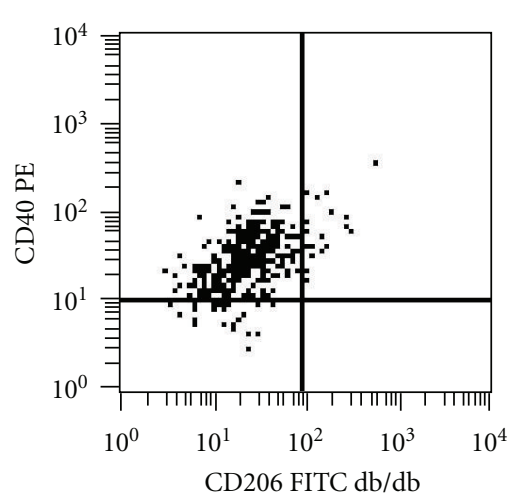

(b)

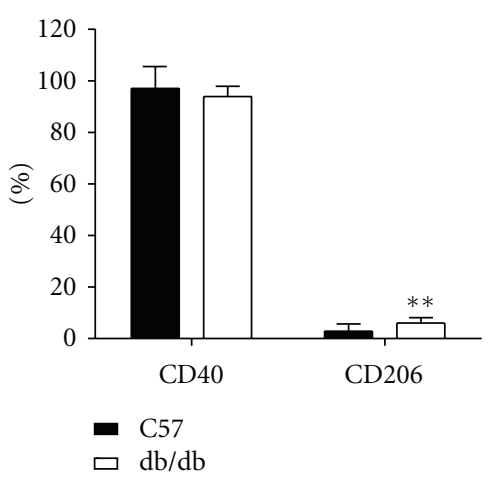

(c)

FIGURE 4: Increased percentage of M2 macrophages in RPMs from db/db mice. One representative example of four independent experiments with identical results is shown. (a) and (b) were RPMs from normal and db/db mice, respectively. And the average percentages of M1 and M2 macrophages were quantified (c). ${ }^{* *} P<0.01$ versus control group.

higher than that of control mice, and the percentage of M1 ( $\mathrm{F} 4 / 80^{+} / \mathrm{CD} 40^{+} / \mathrm{CD} 206^{-}$) macrophages of RPMs in $\mathrm{db} / \mathrm{db}$ mice reduced accordingly.

\section{Discussion}

In the present study, we explored the pathogenesis of diabetes mellitus (DM) with the classical type 2 diabetic mouse model. It was demonstrated in our study that long duration of T2D significantly altered cell numbers, morphology, phenotypes, and functions of resident peritoneal macrophages.

The severe complications of diabetes including impaired wound healing and accelerated atherosclerosis may increase patient's susceptibility to infection and make the infected tissue difficult to be repaired [27]. It was well accepted that the dysfunction of the macrophage system may contribute to these complications. Macrophages play important roles in innate immunity, which help host to defense microbial invaders through producing various cytokines and phagocytosis.

In our study, it was found that the cell numbers of $\mathrm{F} 4 / 80^{+} \mathrm{RPMs}$ in each 5-month-old $\mathrm{db} / \mathrm{db}$ mouse increased obviously, which was contradicted with a previous study performed in STZ-induced type 1 diabetic (T1D) mice without standardization of mouse's body weight. Ma et al. presumed that the reduction of $\mathrm{F} 4 / 80^{+} \mathrm{RPMs}$ in T1D mice was probably related with shortened life span of macrophages and/or the blocking differentiation of macrophages from monocytes or bone marrow cells (BMCs) [20]. Another preliminary study in vitro also showed that only a low level of macrophages was generated in NOD BMCs after being stimulated with GM-CSF [28]. Interestingly, when the number of F4 $/ 80^{+}$RPMs with mouse's body weight was standardized, the result was reversed. As $\mathrm{db} / \mathrm{db}$ mice are larger while STZ-induced T1D mice are much smaller than control, the number of $\mathrm{F} 4 / 80^{+}$RPMs from each mouse may be positively correlated with animal body weight. Nevertheless, as it is well accepted that diabetes and obesity lead to a chronic lowgrade inflammation, diabetes-associated inflammation is correlated with elevated macrophage cell numbers. Previous report about the percentage of bone marrow-derived cells expressing the macrophage marker $\mathrm{F} 4 / 80\left(\mathrm{~F} 4 / 80^{+}\right)$in perigonadal, perirenal, mesenteric, and subcutaneous adipose tissue by immunohistochemical analysis revealed that it was significantly and positively correlated with both adipocyte size and body mass [29]. However, the specific mechanism of different macrophage distribution between adipose tissue and abdominal cavity is still unclear. In the present study, we demonstrated for the first time that the number of RPMs from each mouse was positively correlated with animal body weight.

Furthermore, we were the first to find that the RPMs from $\mathrm{db} / \mathrm{db}$ mice were nearly round in shape as compared with those of control mice with spindle-shaped or irregular morphology. It is not surprising that macrophage heterogeneity presents in different organs or tissues, as well as even in a single organ [30]. Recent studies showed that macrophage phenotypic and functional heterogeneity might be related to a wide diversity of factors, including different precursor cells, different differentiation stages, different microenvironments, or even different stimulatory factors [31]. The response of $\mathrm{F} 4 / 80^{+}$peritoneal macrophages from STZ-induced diabetic mouse at 4 months and control mouse to IFN- $\gamma$ and LPS was also different, which demonstrated that macrophages from diabetic mice produced much less TNF- $\alpha$ and IL- 6 than those of control mice through AKTmTOR and ERK pathways [32]. Hence, this morphologic heterogeneity in RPMs between $\mathrm{db} / \mathrm{db}$ and control mice may hint at the discrepancy of cell physiological functions; the potential mechanism might be related with the alteration of microenvironments, levels of blood glucose, dyslipidemia, and advanced glycation end products.

Macrophages can engulf foreign pathogens, microorganisms and dead cells, which are essential steps of cellular immunity and a part of innate immunity. It was found that phagocytic capacity of F4/80 ${ }^{+}$RPMs from 5 -month-old diabetic mice was lower than that of control with method of detecting the amount of phagocytosis fluorescence-modified 
polystyrene latex beads by FCM. The decreased nonspecific phagocytosis ability of macrophages in diabetic hosts was consistent with several reports about the diabetic host with increased susceptibility to microorganism infection $[33,34]$. Furthermore, remarkably impaired adhesion ability of RPMs was also observed in $\mathrm{db} / \mathrm{db}$ mice. Attenuated adhesion ability in RPMs from $\mathrm{db} / \mathrm{db}$ mice may also be responsible for the dysfunction immunity though this test could not completely mimic the process of macrophages adhesion to endothelial cells in vivo as a first step in the inflammation reaction. However, the mechanisms of macrophages from diabetic hosts with inhibited adhesion capacity need further study. Lastly, the chemotaxis vitality of RPMs from $\mathrm{db} / \mathrm{db}$ mice was also found to be impaired although no significant difference between the two groups was identified. Because the strong relationship between the adipose tissue macrophage content and indicators of adiposity in diabetic mouse provides a mechanism for the increased adipose tissue production of proinflammatory molecules and acute phase proteins, such as TNF- $\alpha$, IL6, and MCP-1 [35]. And increased adipocyte volume and number in diabetic mice are also positively correlated with leptin production, while negatively correlated with production of adiponectin $[36,37]$. As it is well accepted that huge array of environmental factors (including cytokines, chemokines, pattern recognition receptors, and hormones) differentially regulates macrophage response [38], in our study the decreased nonspecific phagocytosis, adhesion, and chemotaxis ability of RPMs in diabetic hosts may be related to the abnormal microenvironment in $\mathrm{db} / \mathrm{db}$ mouse. On the other hand, these findings support the possibility that interrupted wound healing, and complications associated with inflammatory in DM patients, at least in part, is due to the dysfunction of macrophages in phagocytosis, adhesion, and chemotaxis.

During enhanced recruitment in response to disease states, inflammatory monocytes are recruited in response to cytokine cues and undergo differentiation into two broad but distinct subsets of macrophages that are categorized as either classically activated (M1) or alternatively activated (M2). The expression of costimulatory molecules such as CD80, CD86, CD40, and CD54 on macrophages after activation is critically related to the efficient promotion of adaptive immunity by macrophages [39]. CD206 expression is a specific marker for IL-4-induced M2 macrophages, which was used by C. Sun as the M2 marker in a flow cytometry analysis [32]. Hence, we selected the markers CD40 and CD206 as M1 and M2 macrophages, respectively, for our study. Our result showed the proportion of anti-inflammatory M2type RPMs was doubled in $\mathrm{db} / \mathrm{db}$ group compared with the control group. This result was similar with another study in vitro, which showed that markers on peritoneal macrophages isolated from STZ-induced diabetes mice at 4 months after activation were significantly lower compared to control mice as determined by the percentage of positive cells and MFI of CD86 and CD54 expression; whereas arginase activity, another important marker for alternatively activated macrophages, was significantly higher in peritoneal macrophages isolated from STZ-induced diabetes mice than those of control mice when stimulated with IL-4 [32].

Macrophages not only get involved in the etiology of DM and its chronic complications but also defense pathogens, and their proper activated state determines the result of the host response to pathogen aggression. The M1 program of macrophages is usually associated with protection during acute infectious diseases and thought to damage neighboring tissues; M2 macrophages having immunosuppressive and tissue-repairing functions play critical roles in the resolution of harmful inflammation by producing anti-inflammatory mediators $[40,41]$. Therefore, an increased proportion of M2 RPMs in the $\mathrm{db} / \mathrm{db}$ mice may be related with the chronic lowgrade inflammation in abdominal cavity. However, $\mathrm{db} / \mathrm{db}$ mouse is a genetically deficient mouse, and IL-4 signaling in $\mathrm{db} / \mathrm{db}$ macrophages was alleviated compared with $\mathrm{db} /{ }^{+}$or macrophages from control mice, which indicated that the impaired IL-4 pathway in macrophages from the $\mathrm{db} / \mathrm{db}$ mouse model of T2D may induce a state of IL-4 resistance and finally disrupt IL-4-dependent M2 phenotype macrophage yield [42]. This seemingly contradictory result needs further study to explain.

In summary, our study clearly showed that an increased percentage of M2 type RPMs accompanied with remarkable innate immunity dysfunction occurred in type 2 diabetic mice in vivo with a long duration. This study may help us to understand the susceptibility of diabetic patients to infectious diseases and other complications in the patients of diabetes. However, further investigation is needed to clarify the mechanisms involved and possible methods to ameliorate this alteration.

\section{Abbreviations}
AI: $\quad$ Adherence index
CI: Chemotactic index
DM: Diabetic milieu
FMLP: N-formyl-L-methionyl-L-leucyl-L-phenylalanine
FITC: Fluorescein isothiocyanate
FCM: Flow cytometry
MFI: Median fluorescenceintensity
PE: Phycoerythrin
PMNs: Polymorphonuclear neutrophils
RPMs: Resident peritoneal macrophages
T2D: Type 2 diabetes
T1D: Type 1 diabetes.

\section{Acknowledgments}

This study has been funded by Grants to F.-L. Chen from the National Science Foundation of China (30870954), the Foundation for Science and Technology, Shanghai Jiao tong University School of Medicine (YZ1054), the talent project funded by Shanghai Jiao tong University School of Medicine (09XBR01), and the fund for Science and Technology from Third People's Hospital affiliated to Shanghai Jiao tong University Medicine of School (syz2010-02). 


\section{References}

[1] J. M. Fernández-Real and J. C. Pickup, "Innate immunity, insulin resistance and type 2 diabetes," Diabetologia, vol. 55, no. 2, pp. 273-278, 2012.

[2] G. H. Tesch, "Role of macrophages in complications of Type 2 diabetes," Clinical and Experimental Pharmacology and Physiology, vol. 34, no. 10, pp. 1016-1019, 2007.

[3] C. N. Lumeng, J. B. Delproposto, D. J. Westcott, and A. R. Saltiel, "Phenotypic switching of adipose tissue macrophages with obesity is generated by spatiotemporal differences in macrophage subtypes," Diabetes, vol. 57, no. 12, pp. 32393246, 2008.

[4] B. N. Lambrecht, "Alveolar macrophage in the driver's seat," Immunity, vol. 24, no. 4, pp. 366-368, 2006.

[5] F. Y. Chang and M. F. Shaio, "Respiratory burst activity of monocytes from patients with non-insulin-dependent diabetes mellitus," Diabetes Research and Clinical Practice, vol. 29, no. 2, pp. 121-127, 1995.

[6] R. Shurtz-Swirski, S. Sela, A. T. Herskovits et al., "Involvement of peripheral polymorphonuclear leukocytes in oxidative stress and inflammation in type 2 diabetic patients," Diabetes Care, vol. 24, no. 1, pp. 104-110, 2001.

[7] A. Wykretowicz, B. Wierusz-Wysocka, J. Wysocki, A. Szczepanik, and H. Wysocki, "Impairment of the oxygen-dependent microbicidal mechanisms of polymorphonuclear neutrophils in patients with type 2 diabetes is not associated with increased susceptibility to infection," Diabetes Research and Clinical Practice, vol. 19, no. 3, pp. 195-201, 1993.

[8] C. Romano-Carratelli, M. Galdiero, C. Bentivoglio, I. Nuzzo, D. Cozzolino, and R. Torella, "HLA class II antigens and interleukin- 1 in patients affected by type-II diabetes mellitus and hyperlipemia," Journal of Medicine, vol. 24, no. 1, pp. 2834, 1993.

[9] Y. Ohno, N. Aoki, and A. Nishimura, "In vitro production of interleukin-1, interleukin-6, and tumor necrosis factor- $\alpha$ in insulin-dependent diabetes mellitus," Journal of Clinical Endocrinology and Metabolism, vol. 77, no. 4, pp. 1072-1077, 1993.

[10] B. Kulseng, G. Skjåk-BræK, I. Følling, and T. Espevik, "TNF production from peripheral blood mononuclear cells in diabetic patients after stimulation with alginate and lipopolysaccharide," Scandinavian Journal of Immunology, vol. 43, no. 3, pp. 335-340, 1996.

[11] L. Martinez-Pomares, D. M. Reid, G. D. Brown et al., "Analysis of mannose receptor regulation by IL-4, IL-10, and proteolytic processing using novel monoclonal antibodies," Journal of Leukocyte Biology, vol. 73, no. 5, pp. 604-613, 2003.

[12] J. E. Volanakis, "The role of complement in innate and adaptive immunity," Current Topics in Microbiology and Immunology, vol. 266, pp. 41-56, 2002.

[13] S. Gordon, "Alternative activation of macrophages," Nature Reviews Immunology, vol. 3, no. 1, pp. 23-35, 2003.

[14] A. Mantovani, A. Sica, S. Sozzani, P. Allavena, A. Vecchi, and M. Locati, "The chemokine system in diverse forms of macrophage activation and polarization," Trends in Immunology, vol. 25, no. 12, pp. 677-686, 2004.

[15] D. M. Mosser, "The many faces of macrophage activation," Journal of Leukocyte Biology, vol. 73, no. 2, pp. 209-212, 2003.

[16] T. M. B. Rezende, D. L. Vargas, F. P. Cardoso, A. P. R. Sobrinho, and L. Q. Vieira, "Effect of mineral trioxide aggregate on cytokine production by peritoneal macrophages," International Endodontic Journal, vol. 38, no. 12, pp. 896-903, 2005.
[17] A. Mantovani, A. Sica, and M. Locati, "Macrophage polarization comes of age," Immunity, vol. 23, no. 4, pp. 344-346, 2005.

[18] R. M. Steinman and C. L. Moberg, "Zanvil Alexander Cohn 1926-1993," Journal of Experimental Medicine, vol. 179, no. 1, pp. 1-30, 1994.

[19] L. R. Turchyn, T. J. Baginski, R. R. Renkiewicz, C. A. Lesch, and J. L. Mobley, "Phenotypic and functional analysis of murine resident and induced peritoneal macrophages," Comparative Medicine, vol. 57, no. 6, pp. 574-580, 2007.

[20] H. Ma, G. Liu, W. Ding, Y. Wu, L. Cai, and Y. Zhao, "Diabetesinduced alteration of $\mathrm{F} 4 / 80^{+}$macrophages: a study in mice with streptozotocin-induced diabetes for a long term," Journal of Molecular Medicine, vol. 86, no. 4, pp. 391-400, 2008.

[21] Z. A. Cohn and B. Benson, "The differentiation of mononuclear phagocytes. morphology, cytochemistry, and biochemistry," The Journal of Experimental Medicine, vol. 121, pp. 153170, 1965.

[22] W. Z. Ho, J. P. Lai, X. H. Zhu, M. Uvaydova, and S. D. Douglas, "Human monocytes and macrophages express substance P and neurokinin-1 receptor," Journal of Immunology, vol. 159, no. 11, pp. 5654-5660, 1997.

[23] E. E. Bou Ghosn, A. A. Cassado, G. R. Govoni et al., "Two physically, functionally, and developmentally distinct peritoneal macrophage subsets," Proceedings of the National Academy of Sciences of the United States of America, vol. 107, no. 6, pp. 2568-2573, 2010.

[24] K. Imaizumi, T. Kawabe, S. Ichiyama et al., "Enhancement of tumoricidal activity of alveolar macrophages via CD40-CD40 ligand interaction," The American Journal of Physiology, vol. 277, no. 1, part 1, pp. L49-L57, 1999.

[25] C. Yang, J. Y. Zhou, H. J. Zhong et al., "Exogenous norepinephrine correlates with macrophage endoplasmic reticulum stress response in association with XBP-1," Journal of Surgical Research, vol. 168, no. 2, pp. 262-271, 2011.

[26] S. Fujisaka, I. Usui, A. Bukhari et al., "Regulatory mechanisms for adipose tissue M1 and M2 macrophages in diet-induced obese mice," Diabetes, vol. 58, no. 11, pp. 2574-2582, 2009.

[27] B. R. Shah and J. E. Hux, "Quantifying the risk of infectious diseases for people with diabetes," Diabetes Care, vol. 26, no. 2, pp. 510-513, 2003.

[28] T. Nikolic, M. Bunk, H. A. Drexhage, and P. J. M. Leenen, "Bone marrow precursors of nonobese diabetic mice develop into defective macrophage-like dendritic cells in vitro," Journal of Immunology, vol. 173, no. 7, pp. 4342-4351, 2004.

[29] S. P. Weisberg, D. McCann, M. Desai, M. Rosenbaum, R. L. Leibel, and A. W. Ferrante, "Obesity is associated with macrophage accumulation in adipose tissue," Journal of Clinical Investigation, vol. 112, no. 12, pp. 1796-1808, 2003.

[30] L. Monney, C. A. Sabatos, J. L. Gaglia et al., "Th1-specific cell surface protein Tim-3 regulates macrophage activation and severity of an autoimmune disease," Nature, vol. 415, no. 6871, pp. 536-541, 2002.

[31] D. Chakraborty, S. Banerjee, A. Sen, K. K. Banerjee, P. Das, and S. Roy, "Leishmania donovani affects antigen presentation of macrophage by disrupting lipid rafts," Journal of Immunology, vol. 175, no. 5, pp. 3214-3224, 2005.

[32] C. Sun, L. Sun, H. Ma et al., "The phenotype and functional alterations of macrophages in mice with hyperglycemia for long term," Journal of Cellular Physiology, vol. 227, no. 4, pp. 1670-1679, 2012.

[33] M. Ferracini, J. O. Martins, M. R. M. Campos, D. B. C. Anger, and S. Jancar, "Impaired phagocytosis by alveolar 
macrophages from diabetic rats is related to the deficient coupling of LTs to the Fc $\gamma$ R signaling cascade," Molecular Immunology, vol. 47, no. 11-12, pp. 1974-1980, 2010.

[34] B. F. Liu, S. Miyata, H. Kojima et al., "Low phagocytic activity of resident peritoneal macrophages in diabetic mice: Relevance to the formation of advanced glycation end products," Diabetes, vol. 48, no. 10, pp. 2074-2082, 1999.

[35] P. Sartipy and D. J. Loskutoff, "Monocyte chemoattractant protein 1 in obesity and insulin resistance," Proceedings of the National Academy of Sciences of the United States of America, vol. 100, no. 12, pp. 7265-7270, 2003.

[36] Y. Zhang, K. Y. Guo, P. A. Diaz, M. Heo, and R. L. Leibel, "Determinants of leptin gene expression in fat depots of lean mice," The American Journal of Physiology, vol. 282, no. 1, pp. R226-R234, 2002.

[37] E. Tomas, T. S. Tsao, A. K. Saha et al., "Enhanced muscle fat oxidation and glucose transport by ACRP30 globular domain: Acetyl-CoA carboxylase inhibition and AMP-activated protein kinase activation," Proceedings of the National Academy of Sciences of the United States of America, vol. 99, no. 25, pp. 16309-16313, 2002.

[38] R. D. Stout and J. Suttles, "Functional plasticity of macrophages: reversible adaptation to changing microenvironments," Journal of Leukocyte Biology, vol. 76, no. 3, pp. 509-513, 2004.

[39] J. K. Lee, M. H. Ryu, and J. A. Byun, "Immunotoxic effect of $\beta$-chlorolactic acid on murine splenocyte and peritoneal macrophage function in vitro," Toxicology, vol. 210, no. 2-3, pp. 175-187, 2005.

[40] M. Benoit, B. Desnues, and J. L. Mege, "Macrophage polarization in bacterial infections," Journal of Immunology, vol. 181, no. 6, pp. 3733-3739, 2008.

[41] P. J. Murray and T. A. Wynn, "Protective and pathogenic functions of macrophage subsets," Nature Reviews Immunology, vol. 11, no. 11, pp. 723-737, 2011.

[42] J. C. O'Connor, C. L. Sherry, C. B. Guest, and G. G. Freund, "Type 2 diabetes impairs insulin receptor substrate2-mediated phosphatidylinositol 3-kinase activity in primary macrophages to induce a state of cytokine resistance to IL-4 in association with overexpression of suppressor of cytokine signaling-3," Journal of Immunology, vol. 178, no. 11, pp. 68866893, 2007. 


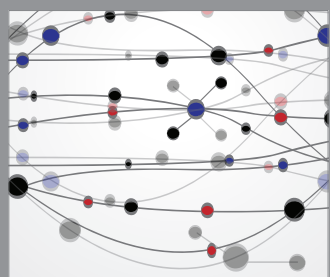

The Scientific World Journal
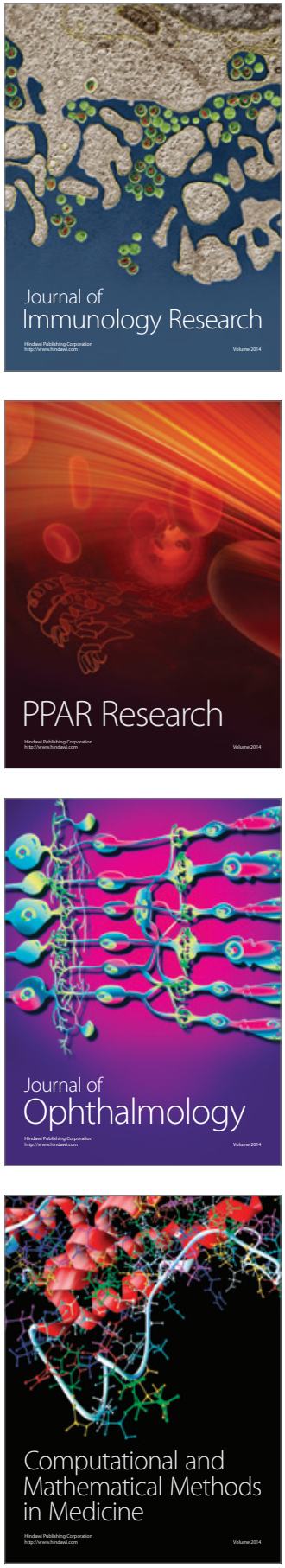

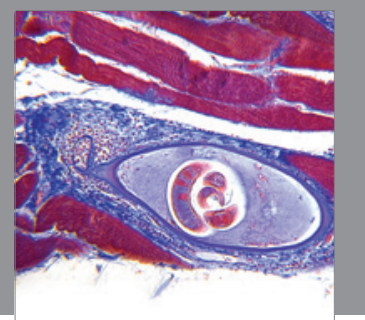

Gastroenterology

Research and Practice
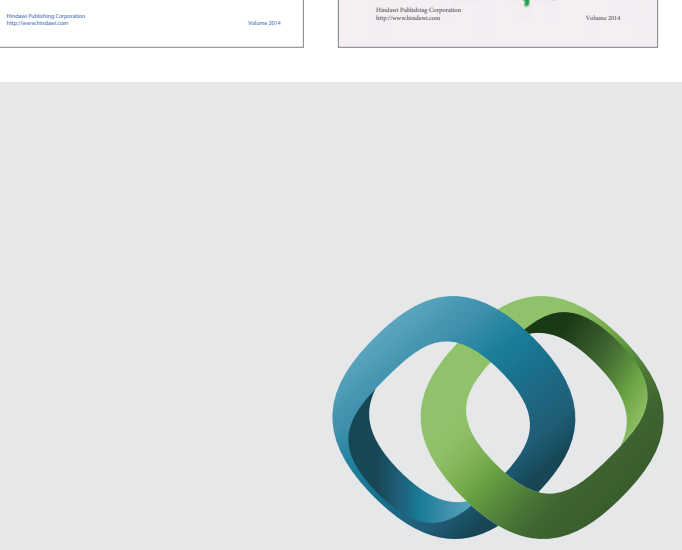

\section{Hindawi}

Submit your manuscripts at

http://www.hindawi.com
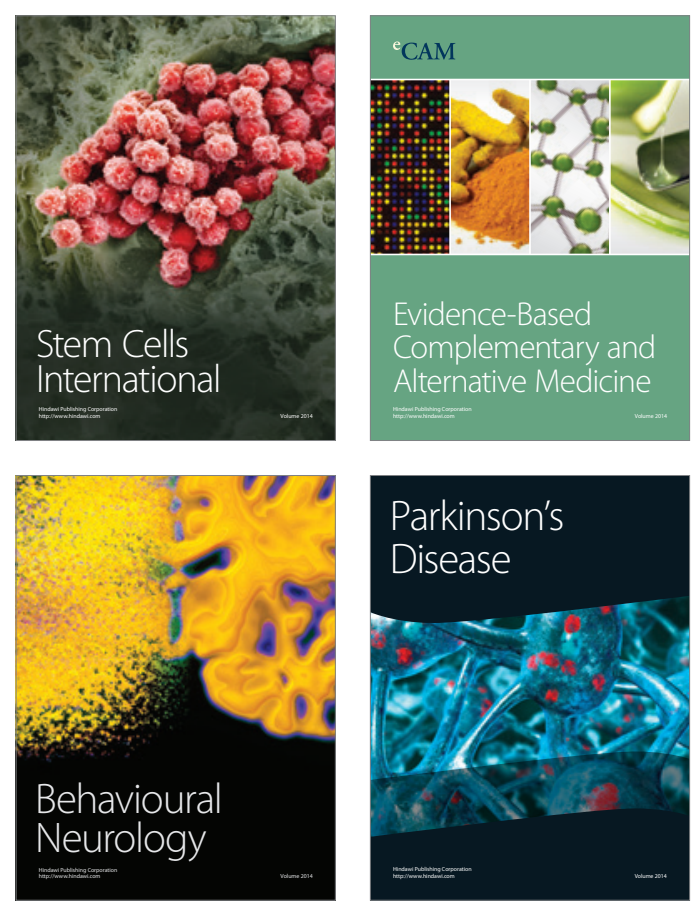

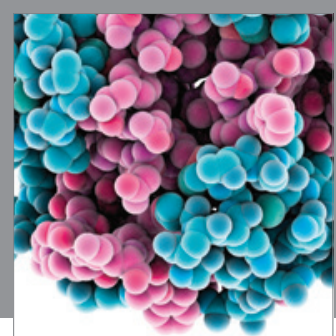

Journal of
Diabetes Research

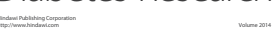

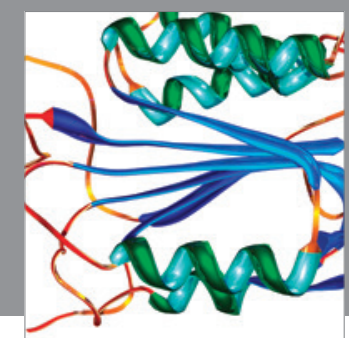

Disease Markers
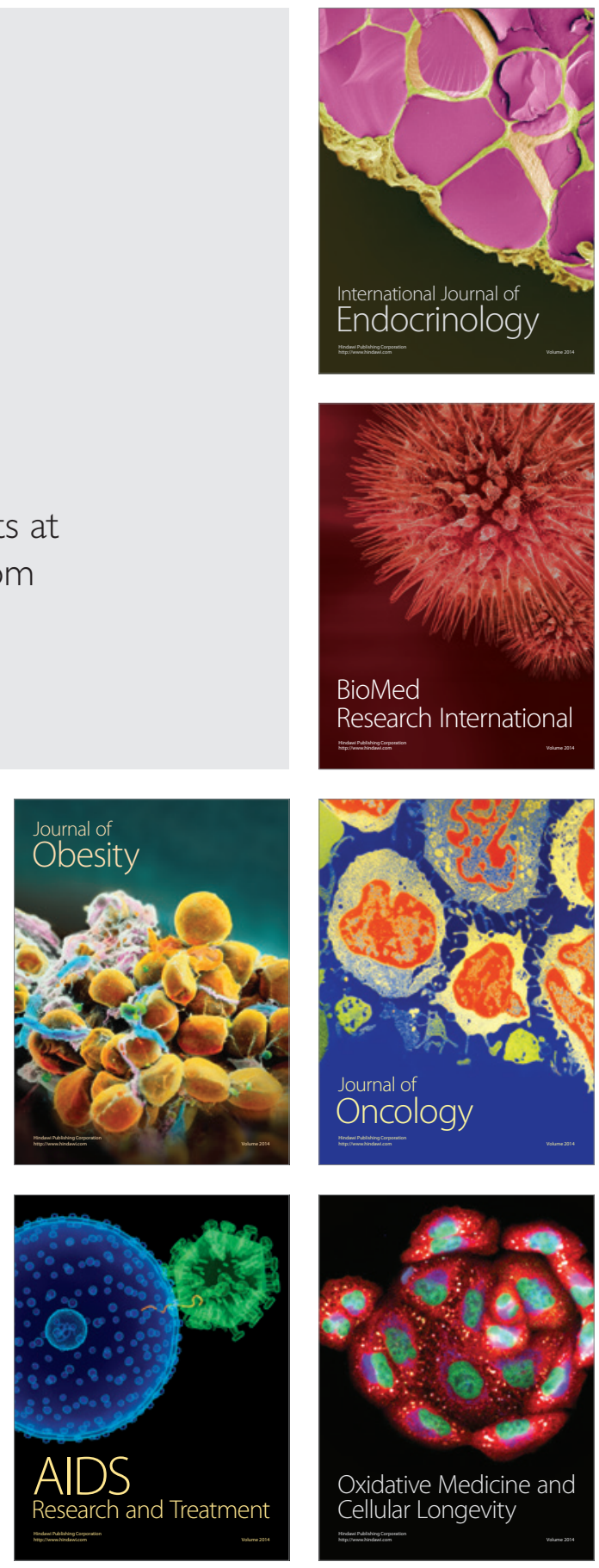